\title{
Stability and change in a perspective reversal illusion
}

\author{
NEIL O. RANKIN \\ Clark University, Worcester, Massachusetts 01610
}

\begin{abstract}
A phenomenological explanation is presented for the stability and instability of the appearance of the form of objects as a consequence of changes in (1) the spatial position of the observer with respect to the object, and (2) the amount of time the object is observed from a specific spatial position. In support of the explanation, an object was constructed so that it would have multiple appearances when observed continuously from the same viewpoint. The initial appearance of this object when observed from different spatial positions and the constancy over time of these appearances were demonstrated to be in agreement with the theoretical expectations.
\end{abstract}

If a straight rod attached to a vertical shaft is rotated at a constant speed and observed continuously at a sufficient distance, it will appear to change its direction of rotation and can be seen at different times as rotating at the same speed, but in different directions. When two people are watching this object from different viewpoints, they will not always agree on the direction of rotation of the object or the position of the object in space. In everyday circumstances, this type of illusion, called a "perspective reversal," can be seen by watching a rotating overhead fan (Kenyon, 1898) or a rotating law'n sprinkler (Miles, 1929). Under laboratory conditions, the illusion has been demonstrated for a variety of objects (Ames, 1951; Day \& Power, 1965; Gillanı, 1972; Pastore, 1952).

These examples of instability in the appearance of objects are of interest not only because of their striking and unusual nature but also because of the challenge they present to psychophysical (Gibson, 1950, 1970; Hochberg \& Brooks, 1960) and "stimulus decoding" (Braunstein, 1972; Johansson, 1950, 1970) theories of perception. The baffling aspect of the phenomenon for these theories is the change in appearance of an object without any change in the temporal pattern of stimulation on the retina. The change in appearance, therefore, cannot be predicted or explained by a change in "information" contained in the stimulation, regardless of how the concept of information is conceived.

Instead of starting the theoretical analysis by taking the energy on the retina as given, the present position begins this analysis by taking an appearance of an object from a specitied spatial position as given. The problem is then to find the principles necessary to determine the stability of this appearance and also the form and stability of other possible appearances from this viewpoint, as well as the stability and form of possible appearances from other spatial viewpoints. ${ }^{1}$

I wish to thank A. Vadum, J. Voneche, and G. Yablick for their help with this research and $S$. Wapner and $M$. Wiener for their critical comments on this paper.
The present experiment investigates the general hypothesis that the stability of an appearance of an object from a particular viewpoint can be understood by considering the geometric relationships between the different possible appearances of the object from that particular viewpoint. In accord with the Gestalt theory of perception (Koftka, 1935) and supported indirectly by research on perspective reversal with drawings of objects (Hochberg \& Brooks, 1960; Kopfermann, 1930; Vitz \& Todd, 1971), two specific hypotheses have been formulated: (1) that the initial appearance of an object will be the appearance having the best organization and (2) that this initial appearance will be stable, i.e., will remain constant over time, to the degree that it is better organized than other possible appearances from the same viewpoint.

Despite the variety of attempts to define the organization (or simplicity) of a form (Attneave, 1954; Hochberg \& Brooks, 1960; Vitz \& Todd, 1971), an exhaustive definition of organization has not yet been formulated which would allow a quantitative comparison of any two forms. However, in many cases, it is possible to order forms by using in combination several known principles of organization. Two of these principles (Koffka, 1935, p. 149-151), (1) the principle of uniformity, which states that a form is organized to the extent that the relationships between its parts are invariant, e.g., the distance and angles between parts being equal and the color being the same in different regions of the figure, and (2) the principle of enclosure, which states that a form is organized to the degree that the parts enclose space, are used in the present study to order the different appearances of one object.

The physical object used in this study was designed so that the expected possible appearances of the object from one viewpoint could be interrelated by simple transformations so that if one of the appearances was specitied the other appearance could be generated by application of the rules of transformation. For this object, the theory predicted that the appearance considered to be better 


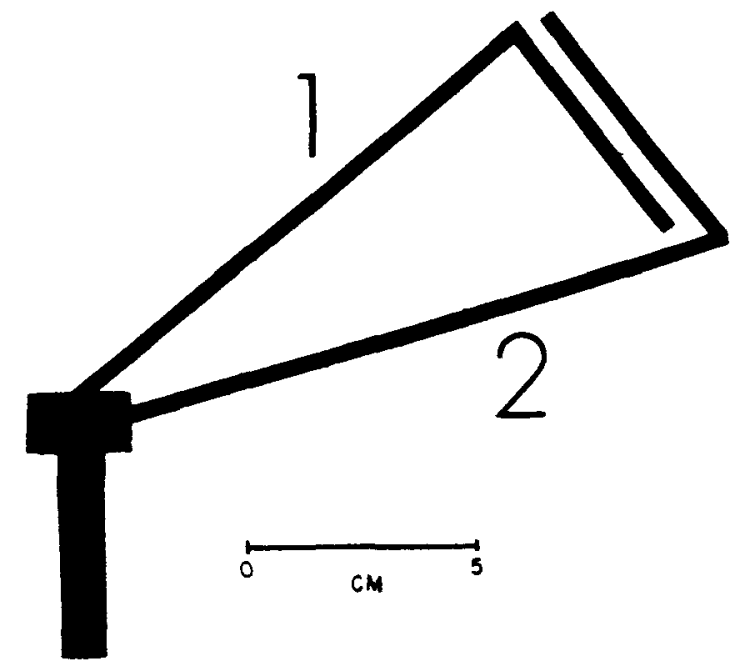

Figure 1. Scale drawing of the object showing Parts 1 and 2.

organized. and consequently the expected initial appearance: (1) would be illusory. (2) the shape of this illusory appearance would be difterent when observed from different viewpoints. and (3) the difference in organization between the expected initial and subsequent appearance. and consequently the stability of the initial appearance. would depend upon the viewpoint from which the physical object was seen.

The object was made of two black wire rods attached to a rertical shaft made of two concentric cylinders and rocated at the same speed but in opposite directions (Figure 1). As riewed from above. the top piece was rotated clockwise and the bottom piece was rotated counterclockwise. It was expected. on the basis of previous work with similar figures (Gillam. 1972: Howard. 1961: Pastore. 1952), that if either part of this object was observed rotating by itselt. at least two appearances would be seen: (1) the part rotating in agreement with the direction of rotation and spatial position of the physical object. and (2) the part rotating at the same speed but in the opposite direction from the physical object and also oriented differently in space. If one appearance was of the part rotating counterclockwise away from the observer. the other appearance would be of the part rotating clockwise toward the observer. The two appearances would have the same orientation only when they were perpendicular to the line of sight.

When both parts of the object are seen simultaneously. the set of expected appearances of the object can be generated from one appearance by systematically transforming both the direction of rotation and orientation of each of the parts. This procedure generates four possible appearances of the object. only one of which has properties in agreement with the phisical object. ${ }^{2}$

These four appearances can be classitied on the basis of their geometric form into two groups, called Forns 1 and $i 1$, where the two members of ach group have the same form but different directions of rotation. Form 1 is seen when the two parts appear to rotate in the same direction. either clockwise or counterclockwise. Form 11 is seen when the two parts appear to rotate in opposite directions. either in agreement with the motion of the physical object or with each part rotating in the direction opposite to its physical motion.

When the two parts are seen rotating in the same direction. the particular shape made by the parts depends upon the viewpoint of the observer with respect to the physical object. Two viewpoints will be considered here: (1) viewing the object from the "tront." Viewpoint $A$, and (2) viewing the object from the "right side." Viewpoint $B$. The parts of the object were positioned so that when seen from Viewpoint $A$. Part 1 would be pointing directly away from the observer when Part 2 was pointing directly tomard the observer. Starting in this position, Figure 2 shows the relative position of the parts of the object as seen trom View points $\mathrm{A}$ and $\mathrm{B}$ as well as the position of the parts in depth as seen from the top. The position of the parts is shown at 45-deg intervals for one complete revolution of each part.

When the physical object is observed from Viewpoint $A$, and the two parts are seen as rotating in the same direction, the object will appear to have a triangular shape that encloses space, with the two parts always appearing directly above and below each other. Form 1A (Figure 2. Viewpoint A). However. when the two parts are seen as rotating in the same

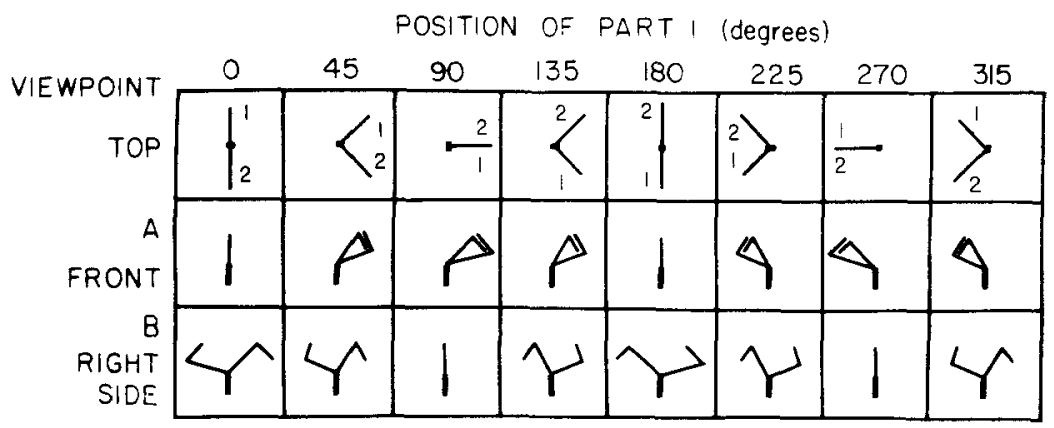

Figure 2. The relative positions of the two parts of the same physical object as seen from three viewpoints at 45 -deg intervals during a complete rotation of each part. Part 1 is rotating clockwise and Part 2 is rotating counterclockwise. 
direction from Viewpoint $\mathbf{B}$, the same physical object will appear to have an open shape, with the two pieces always appearing on directly opposite sides of the vertical shaft, Form $1_{B}$ (Figure 2, Viewpoint $B$ ).

When the physical object is viewed from either viewpoint and the parts appear as rotating in opposite directions, Forms $11_{A}$ and $11_{B}$, the distance between the parts will appear to change continuously and the parts will not appear to have an invariant relationship to each other as they are seen rotating.

The appearances having the forms $1_{\mathrm{A}}, 1_{\mathrm{B}}, 1_{\mathrm{A}}$, and $11_{B}$ differ from each other only with respect to the relationship between the same two parts, and can now be ordered in terms of their degree of organization by the principles of uniformity and enclosure. The best organized form is considered to be $1_{A}$. since the relationship between the parts is invariant as the parts rotate and the parts also enclose space. The next best form would be $1_{B}$, since the relationship between the parts appears invariant but the parts do not enclose space. Forms $11_{\mathrm{A}}$ and $11_{\mathrm{B}}$ have an equal degree of organization that is lower then the degrees of organization of $1_{A}$ and $1_{B}$, since both Forms $11_{A}$ and $11_{B}$ do not have invariant relationships between the parts and the parts also do not enclose space. In decreasing order of organization, then, the forms can be ranked as $1_{\mathrm{A}}$, $1_{\mathrm{B}}$, and $11_{\mathrm{A}}{ }^{-11} \mathrm{~B}$.

Having ordered the appearances according to their degree of organization, the suggested hypotheses may be used to predict (1) the form of the initial appearance of the object from each viewpoint, and (2) the viewpoint where the form of the initial appearance should be more stable. According to the first hypothesis, that the initial appearance will be the appearance having the best possible organization, Form ${ }^{1} A$ should be seen initially from Viewpoint $A$ and Form $1_{B}$ should be initially seen from View point $B$. Both of these forms are illusory and the parts of the object will be seen rotating in the same direction. According to the second hypothesis, that the initial appearance will be stable to the extent that it is better organized than other appearances of the object, the form of the initial appearance from Vieupoint A should be more stable than the form of the initial appearance from Viewpoint $B$, since the difference in degree of organization between $1_{A}$ and $1^{11} \mathrm{~A}$ is larger than the difference in organization between $1_{B}$ and $11_{B}$.

In summary, it is expected that the parts of the object will appear initially to rotate in the same direction from both Viewpoint $A$ and Viewpoint $B$ and that the appearance of the parts rotating in opposite directions will appear sooner from Viewpoint B than from Viewpoint $A$.

\section{METHODS AND RESULTS}

These predictions were tested in an experiment with
10 adults. Five people observed the object at a distance of $5 \mathrm{~m}$ from $V$ iewpoint $A$ and the other five people observed the same object at the same distance from Viewpoint B. The object was presented $35 \mathrm{~cm}$ in front of a translucent screen that was illuminated from behind. The luminance of the surface of the screen facing the observer was $3.4 \mathrm{~cd} / \mathrm{m}^{2}$. Each person observed the object monocularly for $5 \mathrm{~min}$ and was asked to report continuously the apparent direction of rotation for each part separately. Prior to this presentation, each part of the object was shown separately to familiarize the observers with the experinental situation.

The object was made of $.32 \mathrm{~cm}$ in diam wire rods (Figure 1). The longer segment of the top part was $13 \mathrm{~cm}$ long and was mounted at a 42-deg angle to the horizontal. The longer segment of the bottom part was $15 \mathrm{~cm}$ long and was mounted at a 15-deg angle to the horizontal. The two parts of the object were rotated in opposite directions, both at $7 \mathrm{rpm}$.

In accord with the predictions, when the object was seen from either Viewpoint A or Viewpoint B, everyone initially saw the two parts of the object rotating in the same direction. Of the people observing the object from Viewpoint $A$, no one ever saw the parts rotating in opposite directions during the 5 min of observation. However, of the people observing the object from Viewpoint B, everyone saw the parts rotating in opposite directions during the period of observation. For these people, the time to the tirst appearance of opposite rotation ranged from $30 \mathrm{sec}$ to almost $3 \mathrm{~min}$, with a median value over $1 \mathrm{~min}$. The difference in the proportion of people seeing the parts rotating in opposite directions from View point $A$ as compared to Viewpoint $B, 0.0$ vs. 1.0 , is significant at the .01 level by Fisher's exact test.

After the first time the parts were seen rotating in opposite directions from Viewpoint $\mathrm{B}$, some subjects saw the parts of the object change back and forth between rotating in the same direction and rotating in opposite directions. The number of times this change occurred varied for different observers from 0 to 4 with a median value of 1 . The proportion of the entire period of observation that the parts were seen rotating in opposite directions varied for different observers from .20 to .78 with a median value of .51 .

\section{DISCUSSION}

These results are in agreement with the expectation that the stability of the appearance of an object from a specific viewpoint is related to the organization of the possible appearances from that viewpoint. From each viewpoint, the initial appearance was the one considered to have the better organization and the initial appearance was more stable from Viewpoint $A$. the viewpoint associated with the larger difference in the degree of organization between the initial appearance and the expected subsequent appearance. 
In contrast to most objects in everyday circumstances, the object studied exhibited an extreme lack of perceived constancy. This object appears to change markedly its geometric properties when observed continuously from one viewpoint, and the initial appearance of the object has different geometric properties when seen from different view points. The present demonstration that both the initial appearance of the object from different viewpoints and the relative stability of these appearances are predictable gives promise that a structural theory of perception may be developed to explain the stability of our everyday perceptual experience.

The major problem facing the development of such a position is to adequately deal with the specification of the degree of organization of a form. In this paper, the forms could be unambiguously ordered by using the principles of uniformity and enclosure. However, examples may be constructed where these principles or other suggested principles come into conflict with each other so that there would be no clear ordering. and consequently no known way to predict the outcome. The solution would seem to require a unitied concept of organization replacing qualitatively different principles like uniformity and enclosure. As mentioned earlier, current attempts at such unified fornulations of organization (or simplicity) have not been sufficiently developed.

\section{REFERENCES}

AmEs, A. Visual perception and the rotating trapezoidal window. Psychological Monographs. 1951. No. 324.

Attneave, F. Some informational aspects of visual perception. Psychological Review. 1954. 61. 183-193.

BARBUT, M. On the meaning of the word "structure" in mathematics. In M. Lane (Ed.). Introduction to structuralism. New York: Basic Books, 1970.

Braunstein, M. L. Perception of rotation in depth: A process model. Psychological Revien', 1972, 79, 510-524.

DAY, R. H., \& POWER, R. P. Apparent reversal (oscillation) of rotary motion in depth. Psychological Review, 1965, 72. 117-127.

GiBson. J. J. The perception of the visual world. Boston: Houghton Miftlin. 1950.

GiBson, J. J. On theories for visual space perception: A reply to Johansson. Scandinavian Joumal of Psychology, 1970. 11. 75-79.

Gillam, B. Perceived common rotary motion of ambiguous stimuli as a criterion of perceptual grouping. Perception \& Psychophysics, 1972, 11, 99-101.

Hochbe RG, J., \& Brooks, V. The psychophysics of torm: Reversible-perspective drawings of spatial objects. American Journal of Psychology. 1960. 73. 337-354.

HowarD, I. P. An investigation of a satiation process in the reversible perspective of revolving skeletal shapes. Quarterly Journal of Experimental Psychology, 1961, 13, 19-33.

Johansson, G. Configurations in event perception. Uppsala: Alnıquist and Wiksell. 1950.

JohansSON. G. On theories for visual space perception: A letter to Gibson. Scandinavian Joumal of Psychology, 1970, 11. 67-74.

KenYon, F. C. A curious optical illusion connected with an electric fan. Science, 1898, 8, 371-372.

KoffKa. K. Principles of Gestalt psychology. New York: Harcourt Brace. 1935.

Kopfermann, H. Psychologische Untersuchungen über die Wirkung zeidimensionälar Darstellungen körperlicher Gebilde. Psychologische Forschung, 1930. 13, 293-364.

Miles. W. Figure for the "Windmill illusion." Joumal of General Psychology, 1929. 2. 143-145.

PAstore, N. Some remarks on the Ames oscillatory effect. Psychological Review, 1952, 59, 319-323.

VITz, P. C., \& Todd, T. C. A model of the perception of simple geometric figures. Psychological Review. 1971, 78. 207-228.

\section{NOTES}

1. This approach is similar to the way in which the structure of space is considered in theoretical physics. For physical theories. the existence of an object with measurable properties from a particular viewpoint is not problematic, but is taken as given. The problem is to find the mathematical structure which interrelates the measurements of the same object from different reference systems. i.e., different spatial and temporal locations.

2. The transformations interrelating these four appearances have the structure of an algebraic Klein group having four elements. The identity transformation would correspond to no change in direction of rotation or orientation, and the other three transformations would change the direction of rotation or orientation of each part separately and both parts together. These transformations form a closed set and can be shown to satisfy the other axioms of a group (Barbut. 1970). The application of this type of structure to other objects having multiple appearances is currently being studied.

(Received for publication September 27, 1974; revision received January 29,1975 